\title{
Potential for precise Unitarity Triangle angles measurements in LHC
}
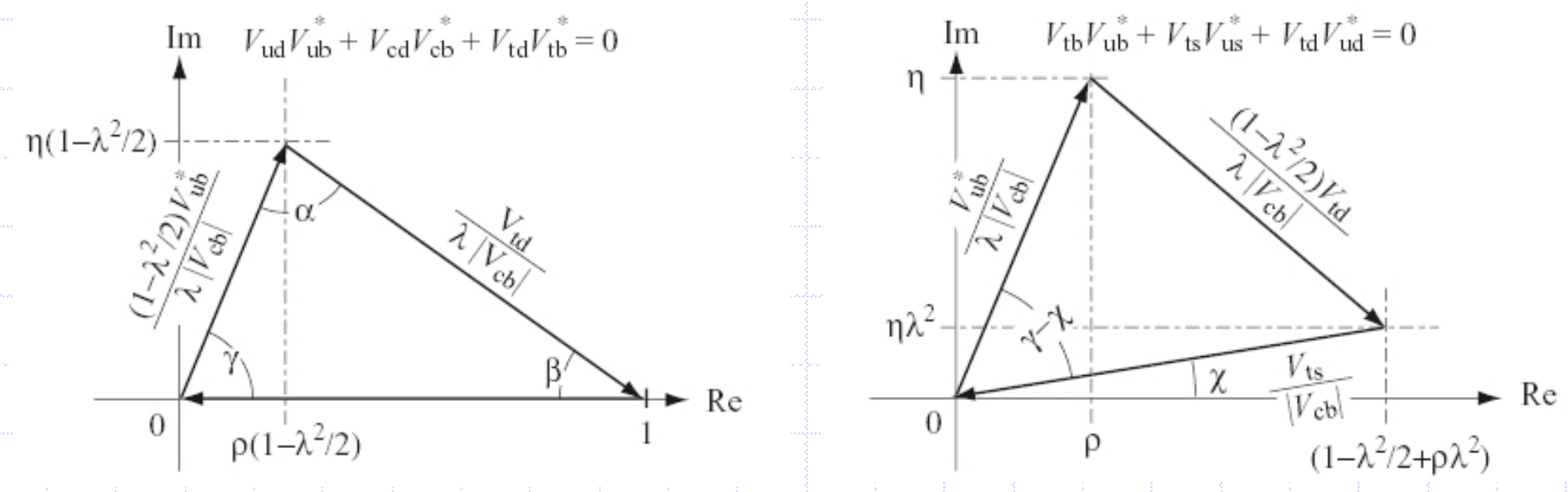

Marco Musy, University of Milano Bicocca 


\section{Motivations}

Current SM fit predicts (including CDF result on $\Delta \mathrm{m}_{\mathrm{s}}$ )

$$
\begin{aligned}
& \alpha=94.6^{\circ} \pm 4.6^{\circ} \\
& \beta=23.9^{\circ} \pm 1.0^{\circ} \\
& \gamma=61.3^{\circ} \pm 4.5^{\circ} \\
& \phi_{s}=2.1^{\circ} \pm 0.2^{\circ}
\end{aligned}
$$

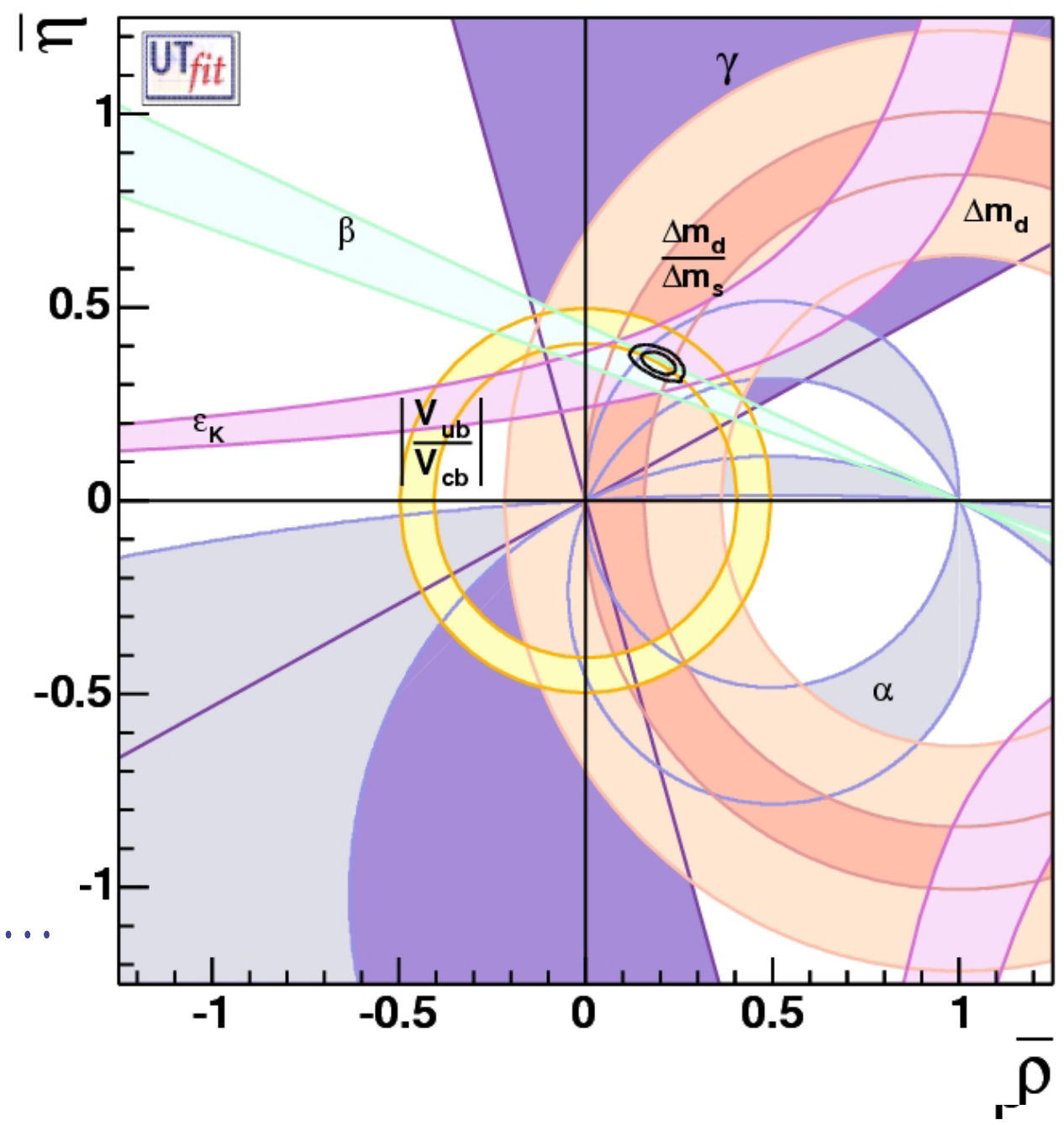

But: New particles may show up in loop diagrams, overconstrain will allow to disentangle SM components from the New Physics ones $\rightarrow$ need very high precision! 


\section{LHC experiments with a $B$ in the menu}
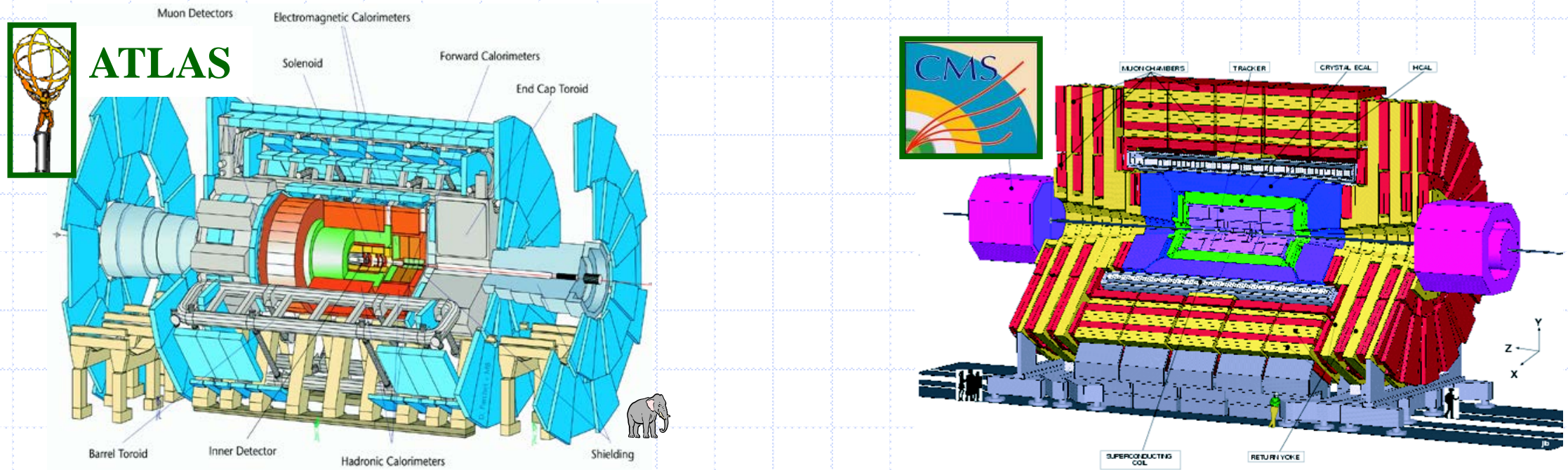

B physics using high- $\mathrm{p}_{T}$ muon, mostly with modes involving dimuons.

B physics dedicated experiment:
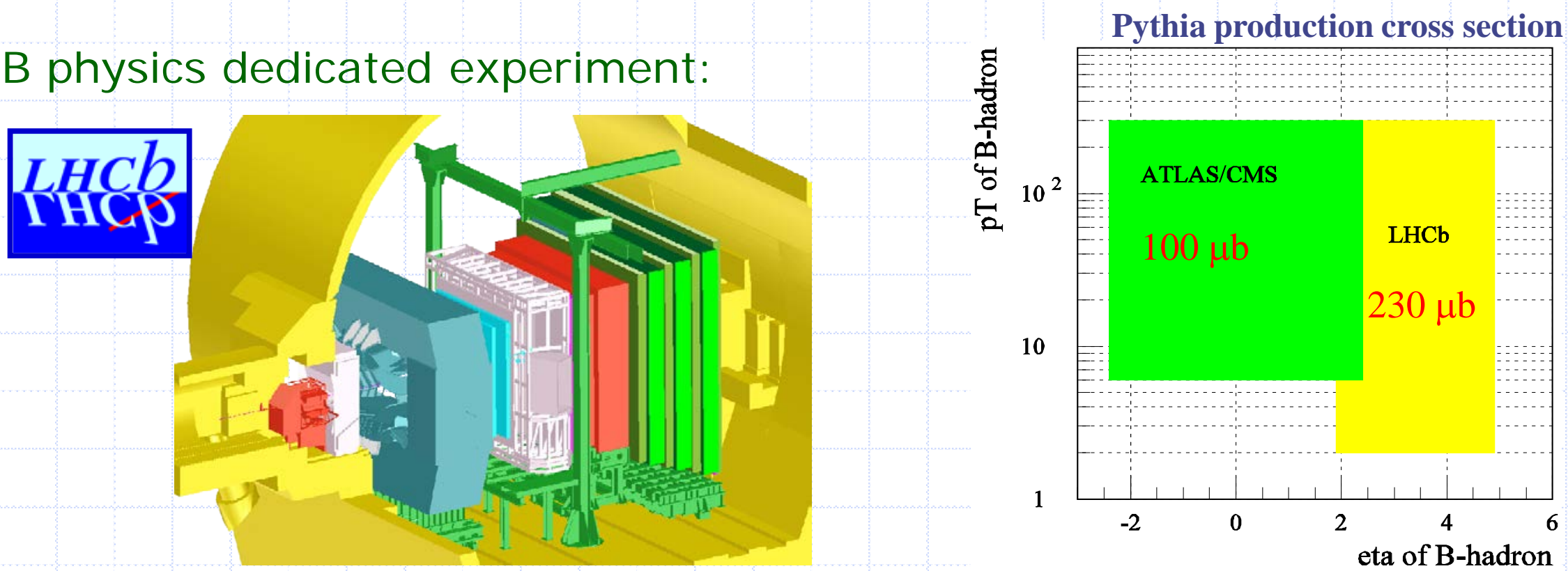


\section{Trigger \& Tagging}

Atlas: B trigger output rate: $10-15 \mathrm{~Hz}$,

Tagging $\varepsilon_{\text {eff }}: \sim 4 \%$

CMS: $5 \mathrm{~Hz}$ inclusive $+\sim 1 \mathrm{~Hz}$ exclusive will both run a few years at $L<2 \times 1033 / \mathrm{cm}^{2} / \mathrm{s}$ with $\mathrm{n}$ (pileup) $<5$, after that $\mathrm{n} \sim 25$

LHCb: $\mathrm{L} \sim 2 \times 10^{32} / \mathrm{cm}^{2} / \mathrm{s}$, n(pileup) 0.5

Tagging $\varepsilon_{\text {eff: }}: 4 \%-5 \%\left(B_{d}\right) 7 \%-9 \%\left(B_{s}\right)$

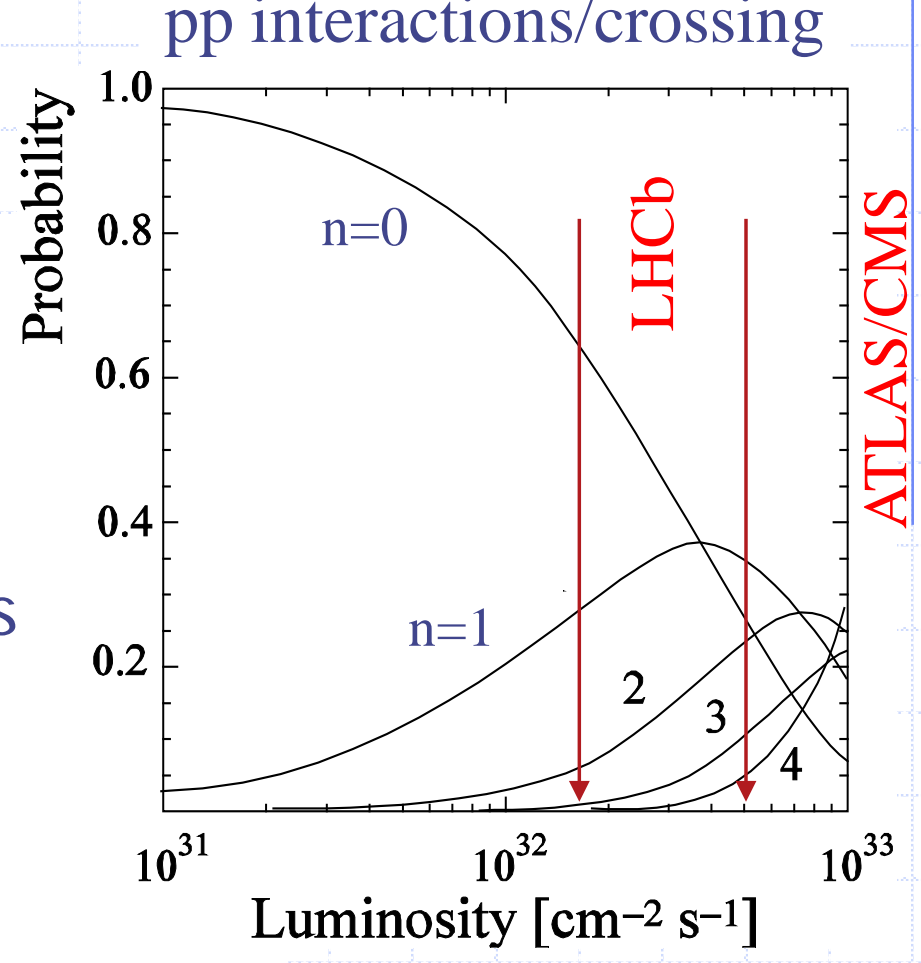

Trigger output:

\begin{tabular}{|c|l|c|c|}
\hline Rate & Event type & Use for physics & $\begin{array}{c}\text { Use for } \\
\text { calibration/ systematics }\end{array}$ \\
\hline $200 \mathrm{~Hz}$ & Exclusive B & $\mathrm{B}$ (core program) & Control channels (tagging, ...) \\
\hline $600 \mathrm{~Hz}$ & High mass dimuon & $130 \mathrm{~Hz}$ of B $\rightarrow J / \psi \mathrm{X}$ & Tracking \\
\hline $300 \mathrm{~Hz}$ & $\mathrm{D}^{*}$ & $65 \mathrm{~Hz} \mathrm{D}^{*}$ (mixing + CPV) & Hadron PID \\
\hline $900 \mathrm{~Hz}$ & Inclusive b (e.g. b $\rightarrow \mu)$ & $550 \mathrm{~Hz}$ of B (data mining) & Trigger \\
\hline
\end{tabular}




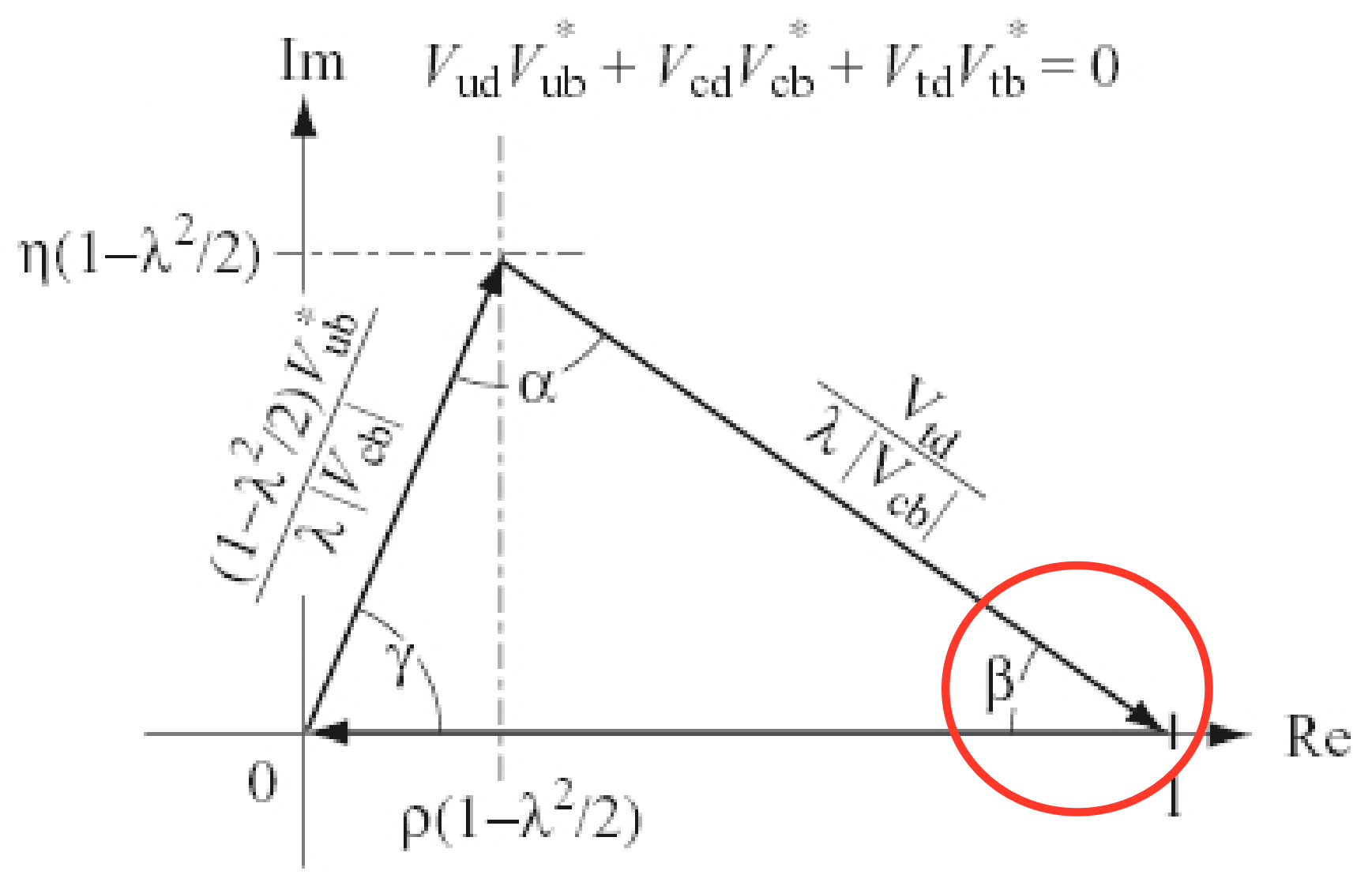




\section{$\boldsymbol{\beta}$ from $B^{0} \rightarrow J / \psi K_{s}$}

$\square$ The 'gold plated' channel at B-factories already well measured by Babar/Belle

- Still an important measurement:

\begin{tabular}{|l|l|}
\hline \multicolumn{2}{|c|}{$A_{C P}^{\text {th }}(t)=A_{C P}^{d i r} \cdot \cos \left(\Delta m_{d} \cdot t\right)+A_{C P}^{m i x} \cdot \sin \left(\Delta m_{d} \cdot t\right)$} \\
$=0$ in SM & $\prod_{=\sin 2 \beta}$
\end{tabular}

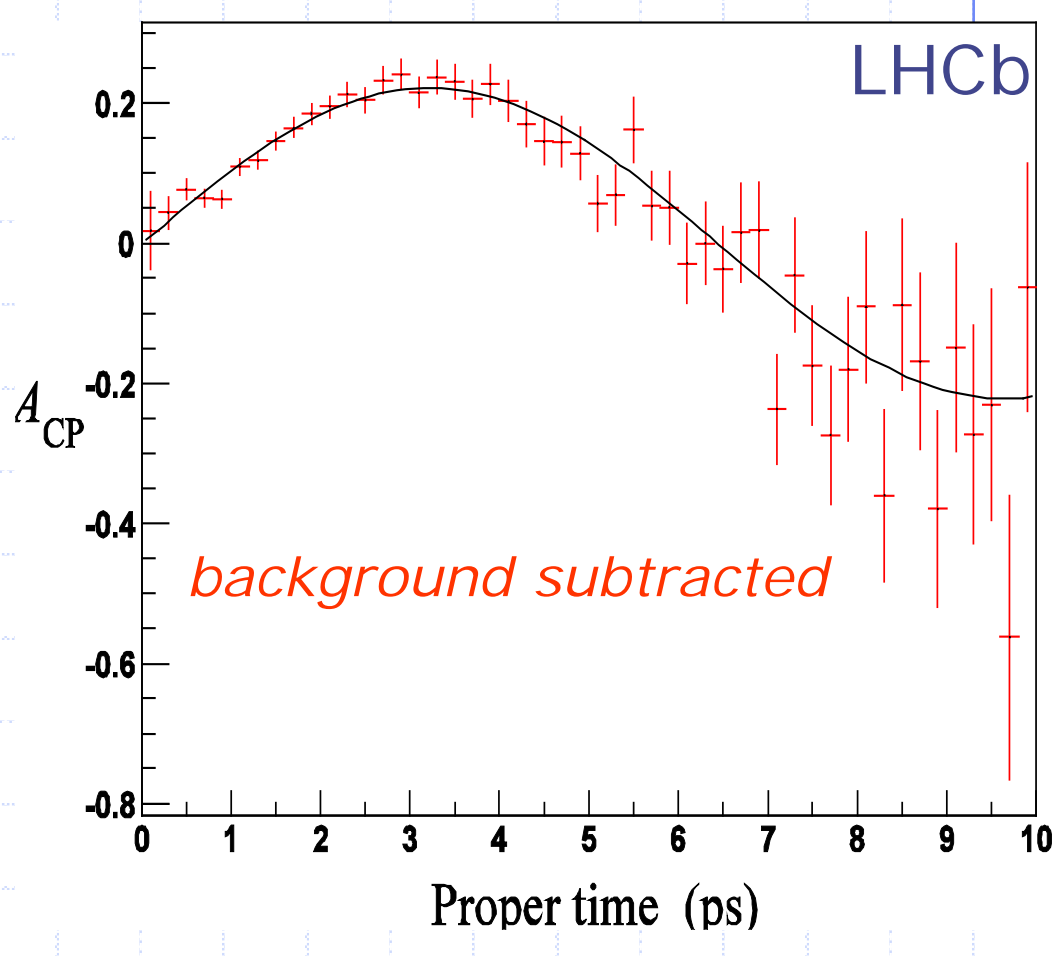

LHCb In one year, $2 / \mathrm{fb}$, with $216 \mathrm{k}$ events, $\sigma(\sin 2 \beta) \sim 0.02, \sigma(\beta) \sim 0.6^{\circ}$

Atlas will achieve similar sensitivity with $30 / \mathrm{fb}$

Comparing with other channels may indicate NP in penguin diagrams

Scaling of 1 year sensitivity from $J / \psi K_{s}$ to $\phi K_{s}$ :

$\sigma\left(\sin 2 \beta_{\text {eff }}\right) \sim 0.4$, Yield: $0.8 \mathrm{k}, \mathrm{B} / \mathrm{S}<2.4$ (preliminary). 


\section{$\phi_{s}$}

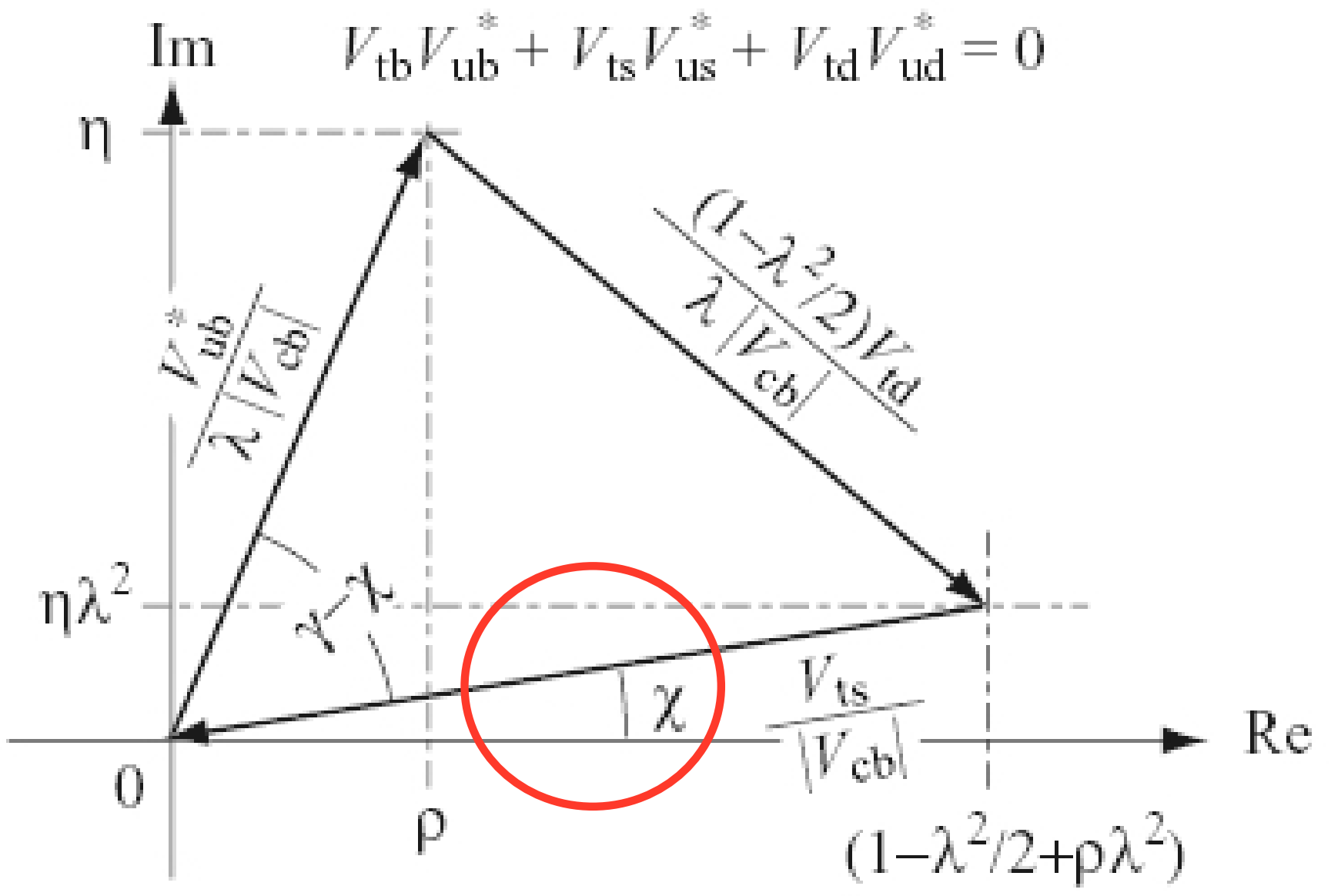




\section{from $B_{s} \rightarrow J / \psi \phi\left(n, n^{\prime} \ldots\right)$}

$\mathrm{B}_{\mathrm{s}} \rightarrow \mathrm{J} / \psi \phi$ is the $\mathrm{B}_{\mathrm{s}}$ counterpart of $\mathrm{B}^{0} \rightarrow J / \psi \mathrm{K}_{\mathrm{S}}$

$\square \operatorname{In~SM~} \phi_{\mathrm{S}}=-2 \arg \left(\mathrm{V}_{\mathrm{ts}}\right)=-2 \lambda^{2} \eta \sim-0.04$

$\square$ Sensitive to New Physics effects in the $B_{s}-\bar{B}_{s}$ system

if NP in mixing $\rightarrow \phi_{S}=\phi_{S}(\mathrm{SM})+\phi_{S}(\mathrm{NP})$

$\square 2$ CP-even, 1 CP-odd amplitudes, angular analysis needed to separate, then fit to $\phi_{S}, \Delta \Gamma_{S}$, CP-odd fraction

\begin{tabular}{|c|c|c|c|c|}
\hline Channels used & $\begin{array}{c}\text { Yield } \\
\left(10^{3} / 2 \mathrm{fb}^{-1}\right)\end{array}$ & $\mathrm{B} / \mathrm{S}$ & $\begin{array}{c}<\delta_{\tau}> \\
(\mathrm{fs})\end{array}$ & $\begin{array}{r}\sigma_{\text {mass }} \\
\left(\mathrm{MeV} / \mathrm{c}^{2}\right)\end{array}$ \\
\hline $\mathbf{B}_{\mathrm{s}} \rightarrow J / \psi\left(\mu^{-} \mu^{+}\right) \phi\left(\mathbf{K}^{+} \mathbf{K}^{-}\right)$ & 131 & 0.12 & 36 & 14 \\
\hline$B_{s} \rightarrow \eta_{c}\left(h^{-} h^{+} h^{-} h^{+}\right) \phi\left(K^{+} K^{-}\right)$ & 3 & 0.6 & 30 & 12 \\
\hline $\mathbf{B}_{\mathbf{s}} \rightarrow \mathbf{J} / \psi\left(\mu^{-} \mu^{+}\right) \eta(\gamma \gamma)$ & 8.5 & 2.0 & 37 & 34 \\
\hline $\mathbf{B}_{\mathbf{S}} \rightarrow \mathbf{J} / \psi\left(\mu^{-} \mu^{+}\right) \eta\left(\pi^{+} \pi^{-} \pi^{0}(\gamma \gamma)\right)$ & 3.0 & 3.0 & 34 & 20 \\
\hline $\mathbf{B}_{\mathbf{s}} \rightarrow \mathbf{J} / \psi\left(\mu^{-} \mu^{+}\right) \eta^{\prime}\left(\pi^{+} \pi^{-} \eta(\gamma \gamma)\right)$ & 2.2 & 2.0 & 32 & 19 \\
\hline$B_{s} \rightarrow D_{s}\left(K^{+} K^{-} \pi^{-}\right) D_{s}\left(K^{+} K^{-} \pi^{+}\right)$ & 4.0 & 0.3 & 56 & 6 \\
\hline
\end{tabular}



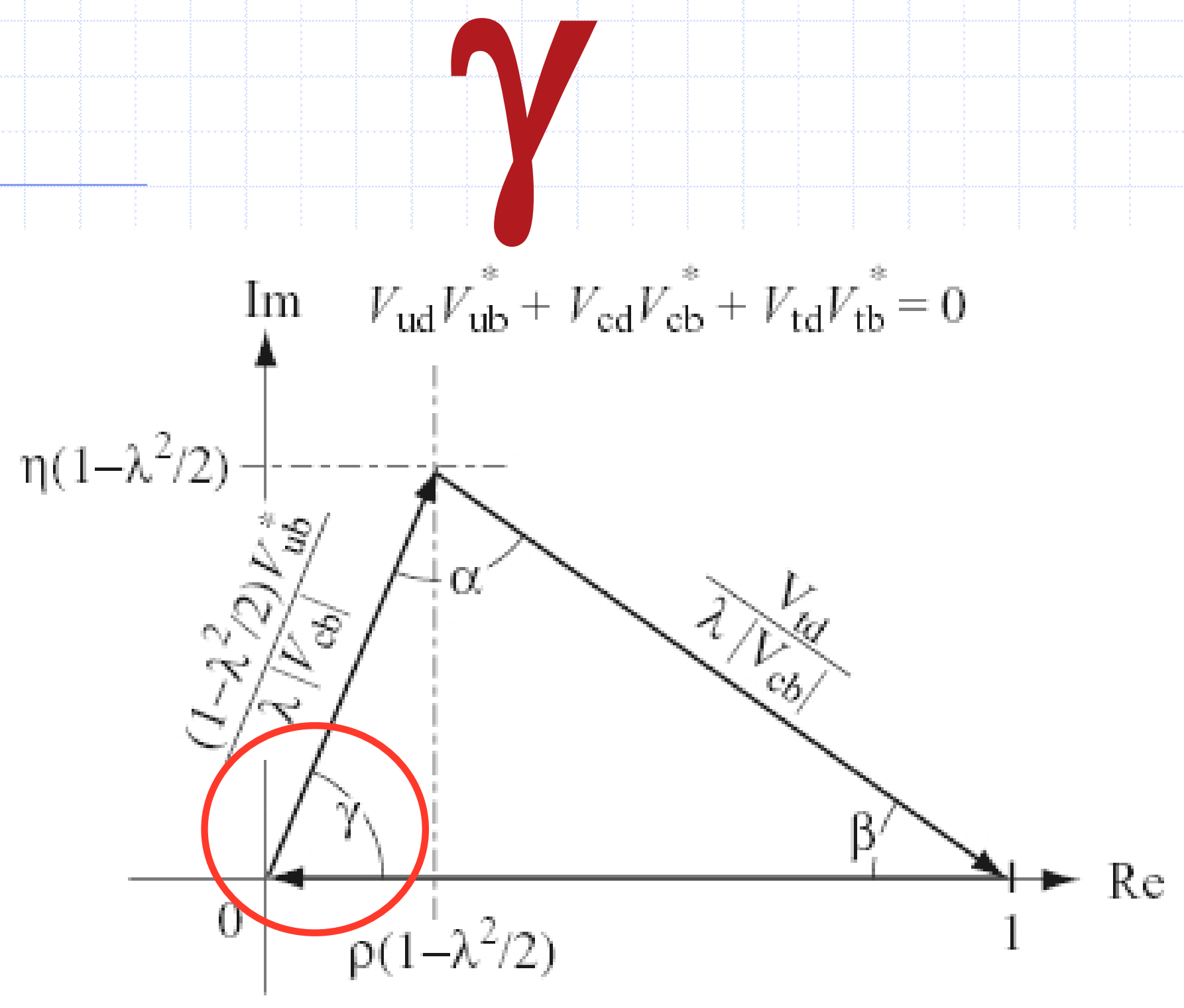


\section{$\gamma$ from $B^{0} \rightarrow D^{0} K^{*}$}

[Phys. Lett. B270, 75 (1991)]
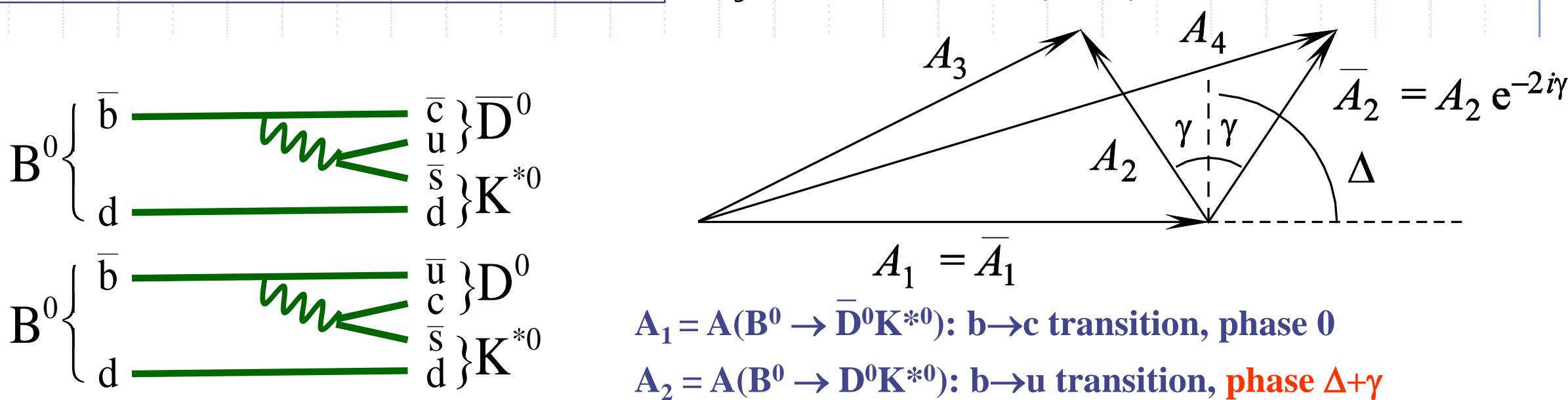

$\mathrm{A}_{1}=\mathrm{A}\left(\mathrm{B}^{0} \rightarrow \overline{\mathrm{D}}^{0} \mathrm{~K}^{* 0}\right): \mathrm{b} \rightarrow \mathrm{c}$ transition, phase 0

$A_{2}=\mathbf{A}\left(B^{0} \rightarrow D^{0} K^{* 0}\right): b \rightarrow u$ transition, phase $\Delta^{+} \gamma$

$A_{3}=\sqrt{ } 2 A\left(B^{0} \rightarrow D_{C P} K^{* 0}\right)=A_{1}+A_{2}$, because $D_{C P}=\left(\bar{D}^{0}+D^{0}\right) / \sqrt{2}$

- Measuring 6 decay rates (self-tagged and time-integrated) allows extraction of $\gamma$

Modes (+CP conj.)

$\mathrm{B}^{0} \rightarrow \mathrm{D}^{0}\left(\mathrm{~K}^{+} \pi^{-}\right) \mathrm{K}^{* 0}\left(\mathrm{~K}^{+} \pi^{-}\right)$

$\mathrm{LHCb}$

$$
\mathrm{B}^{0} \rightarrow \mathrm{D}^{0}\left(\mathrm{~K}^{-} \pi^{+}\right) \mathrm{K}^{\star 0}\left(\mathrm{~K}^{+} \pi^{-}\right)
$$$$
\mathrm{B}^{0} \rightarrow \mathrm{D}^{0}{ }_{\mathrm{CP}}\left(\mathrm{K}^{+} \mathrm{K}^{-}\right) \mathrm{K}^{* 0}\left(\mathrm{~K}^{+} \pi^{-}\right)
$$

Yield (2/fb) $S / B_{b b}(90 \% C L)$

$3.4 \mathrm{k}$

$>2.0$

$0.5 \mathrm{k}$

$>0.3$

$0.6 \mathrm{k}$

$>0.3$

Precision: $\sigma(\gamma) \sim 8^{\circ}$ (in 1year, $2 / \mathrm{fb}$, for $55^{\circ}<\gamma<105^{\circ},-20^{\circ}<\Delta<20^{\circ}$ ) 


\section{from $B^{ \pm} \rightarrow D^{ \pm}$}

- Measure relative rates of $\mathrm{B}^{-} \rightarrow \mathrm{D}(\mathrm{K} \pi) \mathrm{K}^{-}$and $\mathrm{B}^{+} \rightarrow \mathrm{D}(\mathrm{K} \pi) \mathrm{K}^{+}$

- Two interfering tree B-diagrams, one colour-suppressed $\left(r_{B} \sim 0.077\right)$

- Two interfering tree D-diagrams, one Double Cabibbo-suppressed $\left(r_{\mathrm{D}}{ }^{\mathrm{K} \pi} \sim 0.06\right)$

$\rightarrow$ large interference because of similar amplitudes!
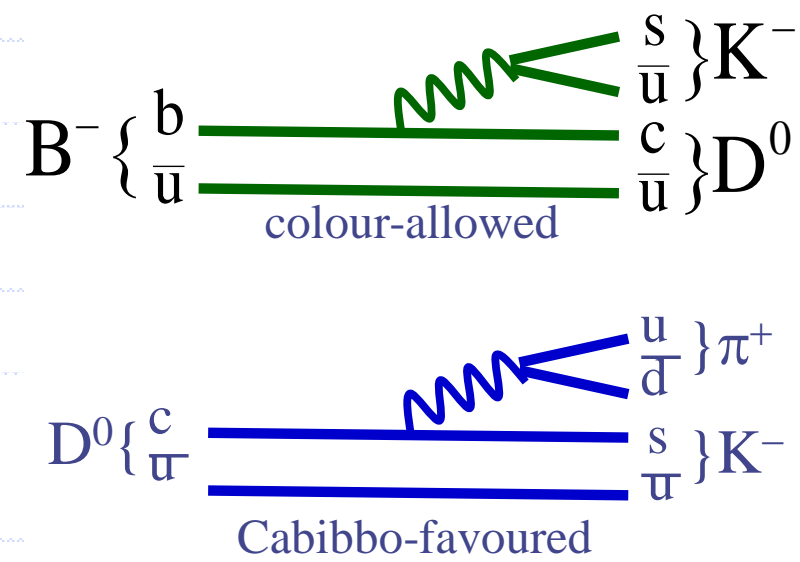
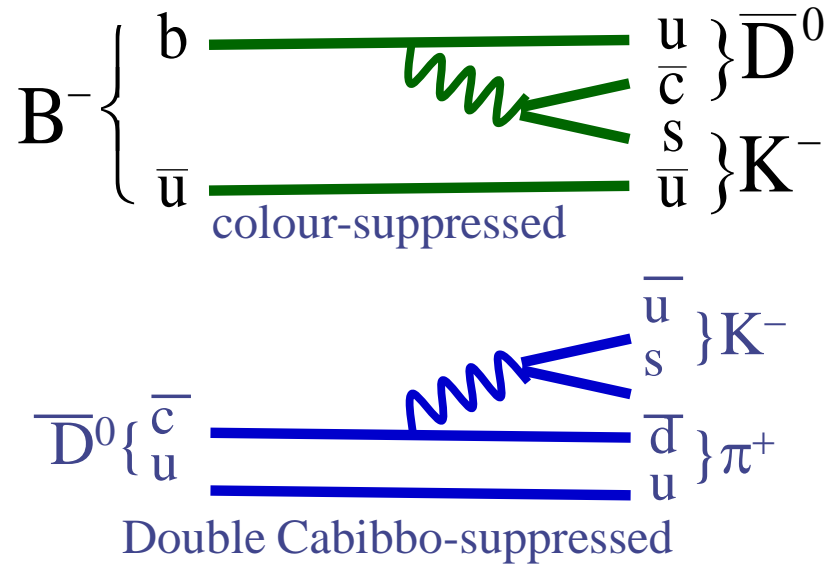

Weak phase diff.: $\quad \gamma$ Magnitude ratio: $\quad r_{B}$ Strong phase diff.: $\delta_{\mathrm{B}}$

Magnitude ratio: $\quad \mathrm{r}_{\mathrm{D}}{ }^{\mathrm{K} \pi}$ Strong phase diff.: $\delta_{\mathrm{D}} \mathrm{K} \pi$

measure:

$$
\begin{aligned}
& \Gamma\left(\mathrm{B}^{-} \rightarrow\left(\mathrm{K}^{-} \pi^{+}\right)_{\mathrm{D}} \mathrm{K}^{-}\right) \propto 1+\left(r_{B} r_{D}^{\mathrm{K} \pi}\right)^{2}+2 r_{B} r_{D}^{\mathrm{K} \pi} \cos \left(\delta_{B}-\delta_{D}^{\mathrm{K} \pi}-\gamma\right) \\
& \Gamma\left(\mathrm{B}^{-} \rightarrow\left(\mathrm{K}^{+} \pi^{-}\right)_{\mathrm{D}} \mathrm{K}^{-}\right) \propto r_{B}^{2}+\left(r_{D}^{K \pi}\right)^{2}+2 r_{B} r_{D}^{K \pi} \cos \left(\delta_{B}+\delta_{D}^{K \pi}-\gamma\right), \\
& \Gamma\left(\mathrm{B}^{+} \rightarrow\left(\mathrm{K}^{+} \pi^{-}\right)_{\mathrm{D}} \mathrm{K}^{+}\right) \propto 1+\left(r_{B} r_{D}^{\mathrm{K} \pi}\right)^{2}+2 r_{B} r_{D}^{\mathrm{K} \pi} \cos \left(\delta_{B}-\delta_{D}^{K \pi}+\gamma\right) \\
& \Gamma\left(\mathrm{B}^{+} \rightarrow\left(\mathrm{K}^{-} \pi^{+}\right)_{\mathrm{D}} \mathrm{K}^{+}\right) \propto r_{B}^{2}+\left(r_{D}^{\mathrm{K} \pi}\right)^{2}+2 r_{B} r_{D}^{\mathrm{K} \pi} \cos \left(\delta_{B}+\delta_{D}^{K \pi}+\gamma\right)
\end{aligned}
$$


$>2$ observables, 5 parameters $\left(\gamma, \delta_{B}, r_{B}, \delta_{D}{ }^{K} \pi, r_{D}{ }^{K} \pi\right), r_{D}{ }^{K} \pi \sim 0.06$ known add more $\mathrm{D}$-decays to constrain further:

$\mathrm{D} \rightarrow \mathrm{K} \pi \pi \pi$ (Cabibbo favoured $+\mathrm{DCS}$ )

$\checkmark 4$ new rates with 2 new parameters, $\delta_{D}{ }^{K} 3 \pi ; r_{D}{ }^{K 3 \pi} \sim 0.06$

$\mathrm{D} \rightarrow \mathrm{KK} \quad$ (CP eigenstate)

$\checkmark 2$ new rates, no new unknown: $\mathrm{r}_{\mathrm{D}}{ }^{\mathrm{KK}}=1 ; \delta_{\mathrm{D}}{ }^{\mathrm{KK}}=0$

$\rightarrow 7$ relative rates and 5 unknowns: $\gamma, \mathrm{r}_{\mathrm{B}}, \delta_{\mathrm{B}}, \delta_{\mathrm{D}}^{\mathrm{K} \pi}, \delta_{\mathrm{D}}^{\mathrm{K} 3 \pi}$ this may come frem-C

Precision: $\sigma(\gamma) \sim 4-13$ in 1 year, $2 / \mathrm{fb}$

depending on $\delta_{\mathrm{D}}^{\mathrm{K} \pi}\left(-25<\delta_{\mathrm{D}}^{\mathrm{K} \pi}<25\right)$

and on $\delta_{\mathrm{D}}{ }^{\mathrm{K} 3 \pi}\left(-180<\delta_{\mathrm{D}}{ }^{\mathrm{K} 3 \pi}<180\right)$

$>$ Extraction of $\gamma$ via Dalitz study $\left(D \rightarrow K_{s} \pi \pi\right)$ is under investigation. 


\section{$\gamma$ from $B \rightarrow \pi \pi, B_{s} \rightarrow K K$}
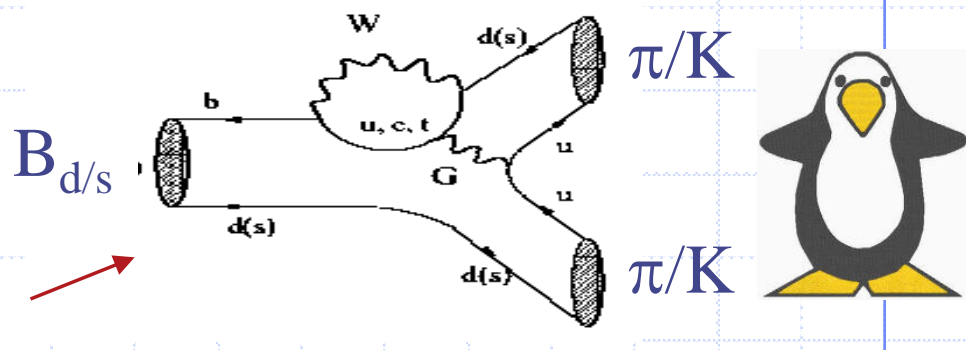

$\square$ Evaluation of $\boldsymbol{A}_{\boldsymbol{C P}}^{\text {dir }}$ and $\boldsymbol{A}_{\boldsymbol{C P}}^{\boldsymbol{m i x}}$ parameters from time-dependent measured asymmetry depend on $\gamma$, mixing phases, and ratio of penguin/tree $=d e^{i \theta}$

$\square$ Assume U-spin symmetry $d_{\pi \pi}=d_{\mathrm{KK}} \theta_{\pi \pi}=\theta_{\mathrm{KK}}$ ( and $\phi_{\mathrm{s}, \mathrm{d}}$ from $\mathrm{B}_{\mathrm{s}} \rightarrow \mathrm{J} / \psi \phi, \mathrm{B} \rightarrow \mathrm{J} / \psi \mathrm{K}_{\mathrm{s}}$ )

$\rightarrow$ solve for $\gamma$

$$
A_{C P}^{t h}(\tau)=\frac{A_{C P}^{d i r} \cdot \cos (x \cdot \tau)+A_{C P}^{m i x} \cdot \sin (x \cdot \tau)}{\cosh \left(\frac{\Delta \Gamma}{2} \cdot \tau\right)-A_{\Delta \Gamma} \cdot \sinh \left(\frac{\Delta \Gamma}{2} \cdot \tau\right)}
$$

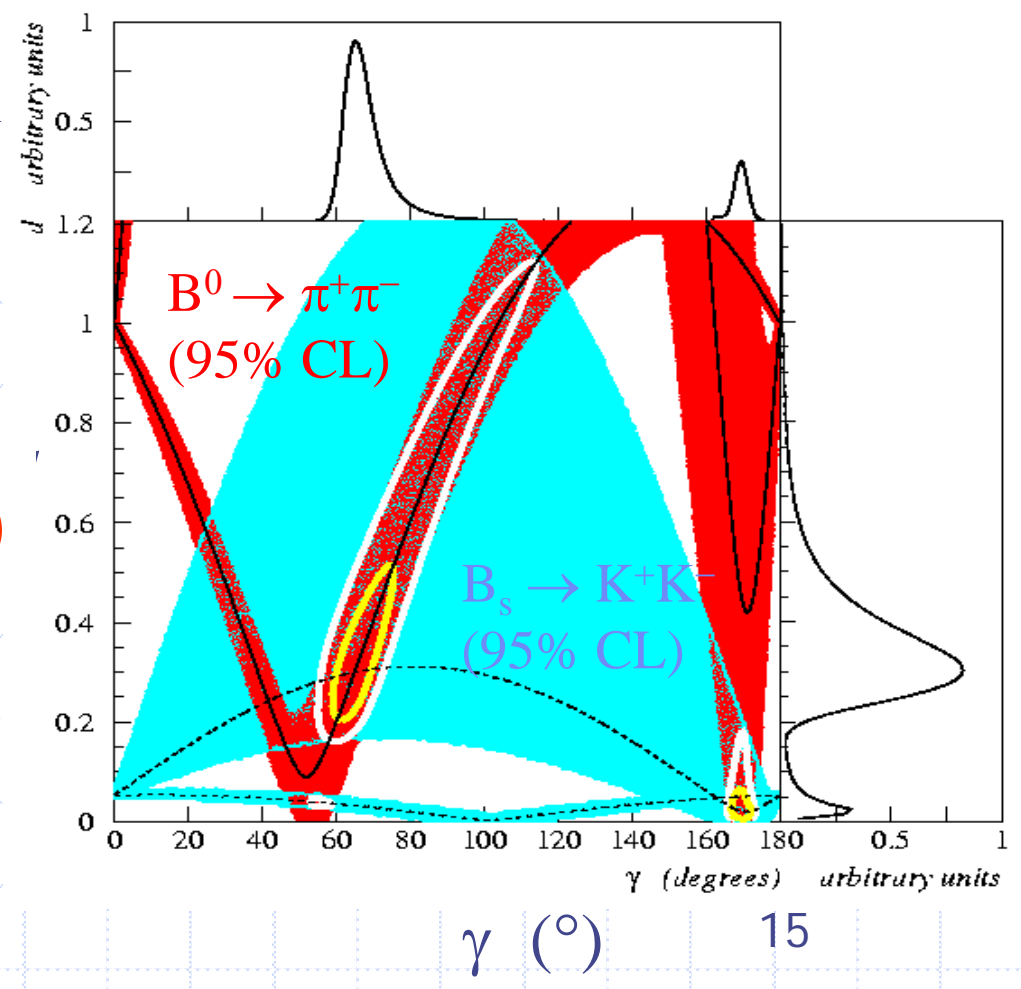




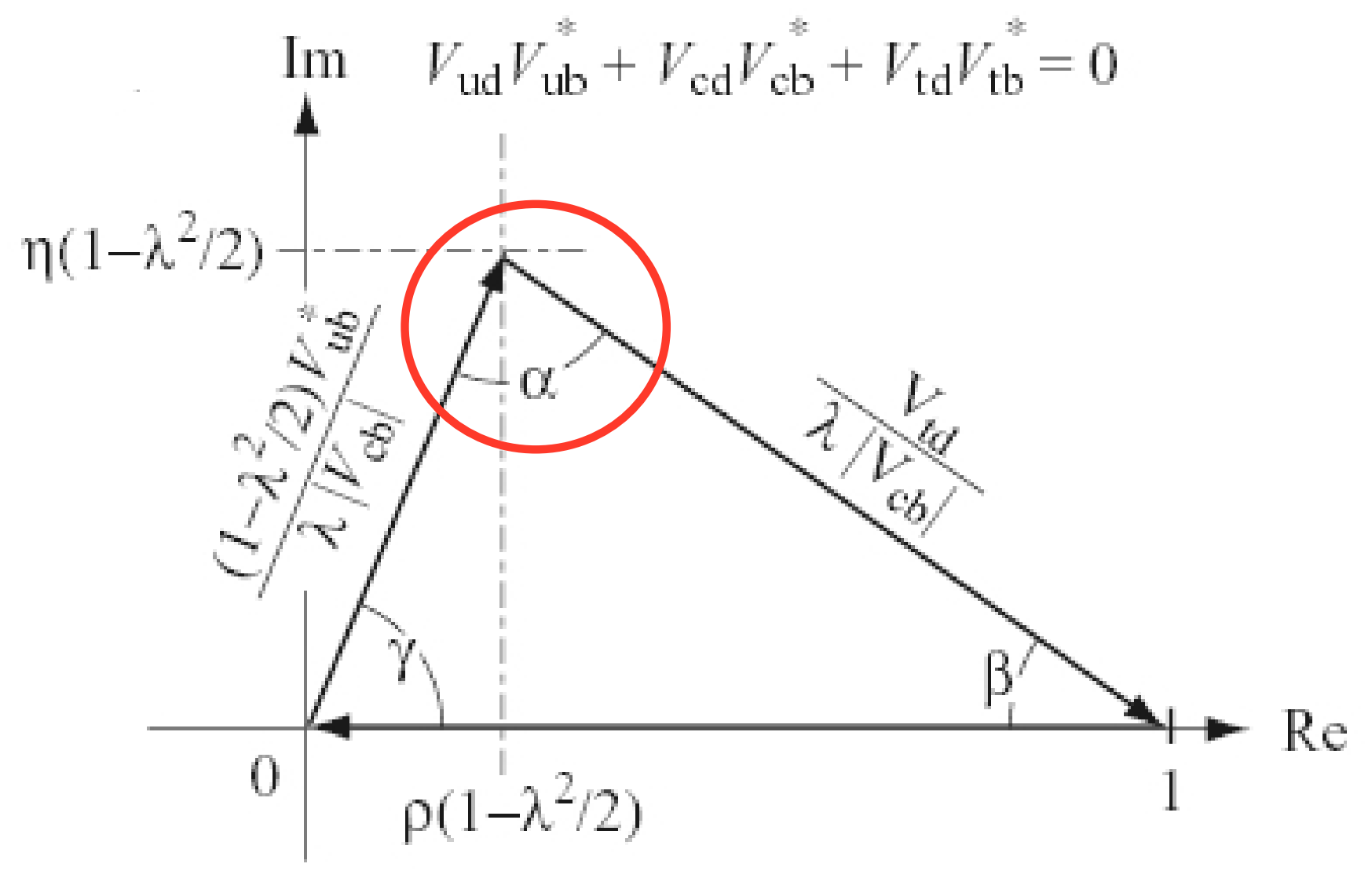




\section{$\boldsymbol{\alpha}$ from $B^{0} \rightarrow \rho \pi$}

Thanks to the interferences between the transitions $B \rightarrow \rho \pi \rightarrow \pi^{-} \pi^{0} \pi^{+}$ we can simultaneously extract $\alpha$ with amplitudes and strong phases from the time dependence of the tagged Dalitz plot

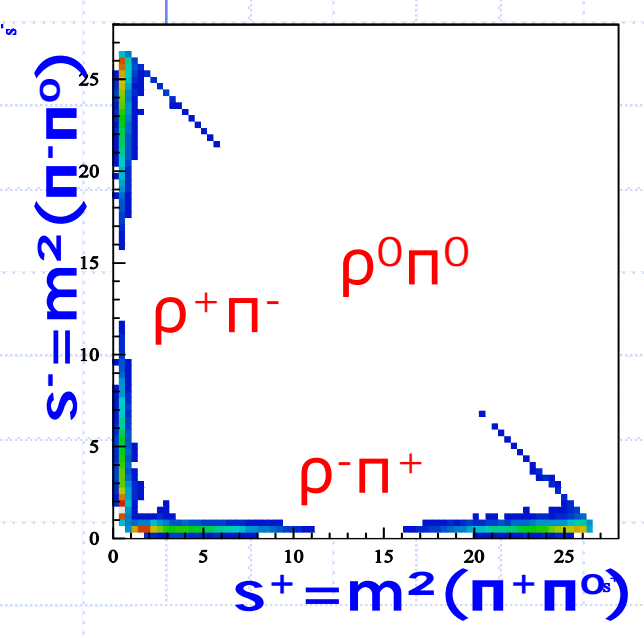

- Simulate the experimental effects: resolution, acceptance, wrong tag, ... Assume $B / S=1$ (mix of flat and resonant $\rho$ )

a Maximize the likelihood wrt $\mathrm{a}^{\text {fit }}$ and the background ratios $r^{\text {fit }}$ (12D fit)
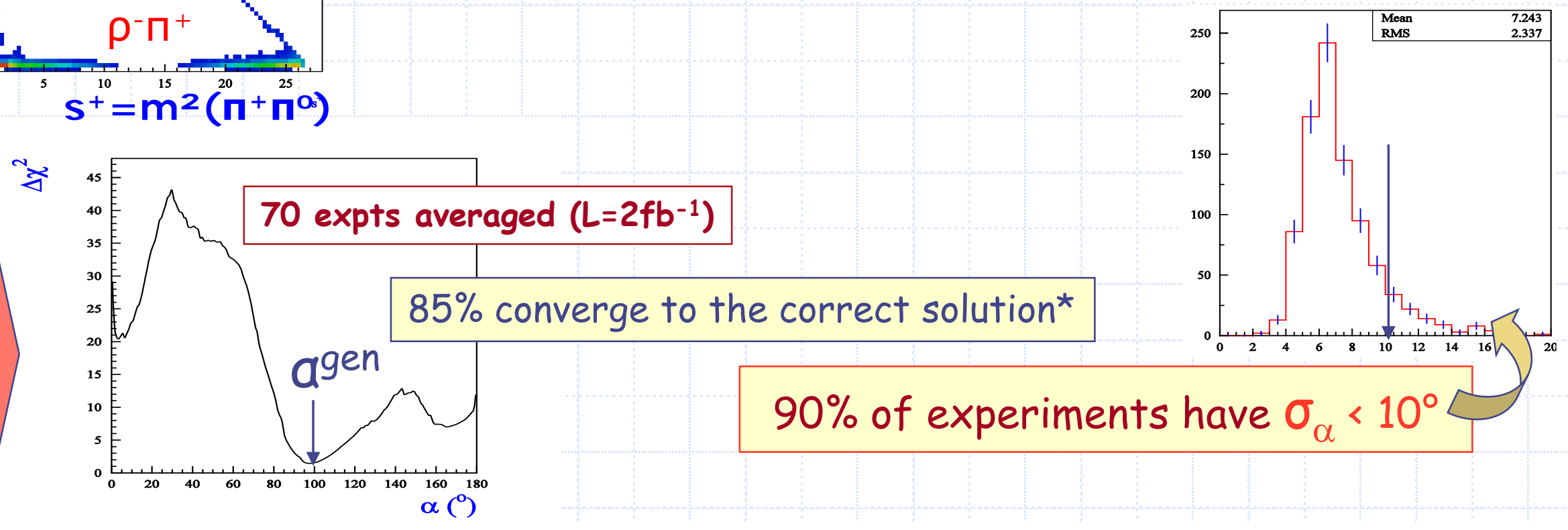

* prob. of mirror solutions decreases with stats, down to $\sim 0.2 \%$ for $10 / \mathrm{fb}$ 


\section{$\boldsymbol{\alpha}$ from $B^{0} \rightarrow \rho \rho$}

Measuring the time dependent asymetry of $B \rightarrow \rho^{+} \rho^{-}$provide $\alpha_{\text {eff }}=\alpha+\Delta \alpha$

$$
\begin{array}{r}
A_{\rho \rho}^{+-}(t)=S_{\rho \rho}^{+-} \sin \left(\Delta m_{d} t\right)-C_{\rho \rho}^{+-} \cos \left(\Delta m_{d} t\right) \\
\text { with } S_{\rho \rho}^{+-}=\sqrt{1-C_{\rho \rho}^{+-2}} \sin \left(2 \alpha_{\text {eff }}\right)
\end{array}
$$

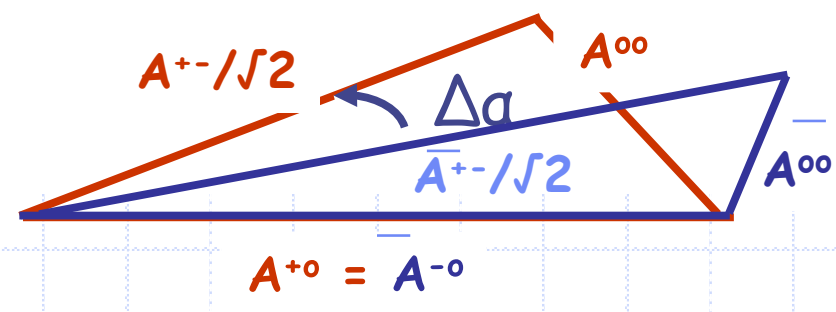

$\mathrm{LHCb}$ is not competitive with current B-factory performance in $\rho^{+} \rho^{-}$. The main contribution of LHCb to the $\rho \rho$ analysis could be the measurement of the $B \rightarrow \rho^{0} \rho^{0}$ mode

Yields in 2/fb:

$B \rightarrow \rho^{+} \rho^{-}: 2 k(B / S<5,90 \% C L)$

$B^{ \pm} \rightarrow \rho^{ \pm} \rho^{0}: 9 \mathrm{k}(\mathrm{B} / \mathrm{S} \sim 1)$

$B \rightarrow \rho^{0} \rho^{0}: \sim 0.5 \mathrm{k}$, assuming a $B R=0.510^{-6}$

(Babar: $\left.B^{00}=\left(0.54_{-0.32}^{+0.36} \pm 0.19\right) 10^{-6}\right)$

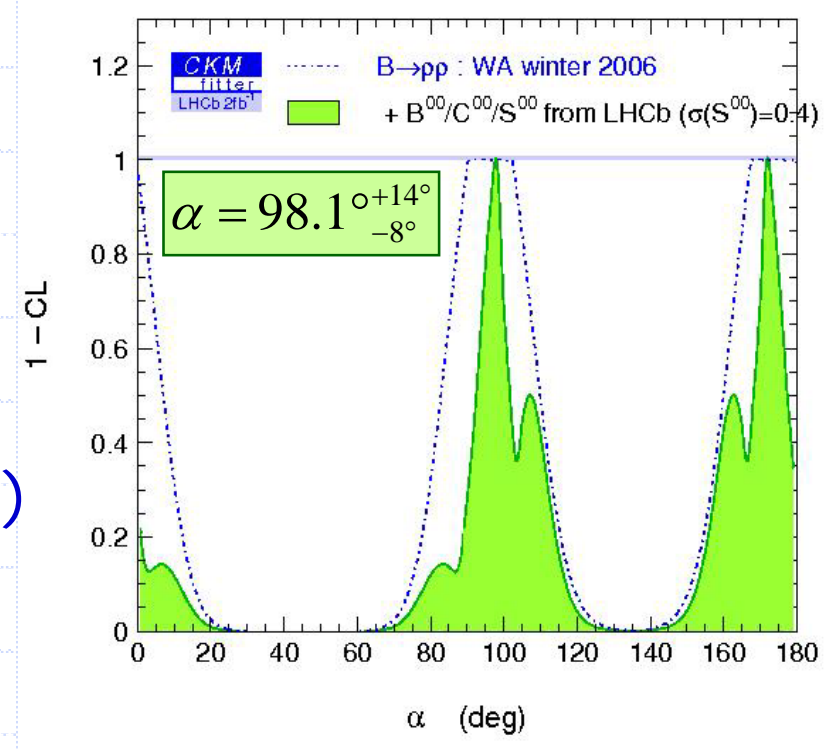




\section{$\boldsymbol{\alpha}$ from $B^{0} \rightarrow \rho \pi$, $\rho \rho$ combined}
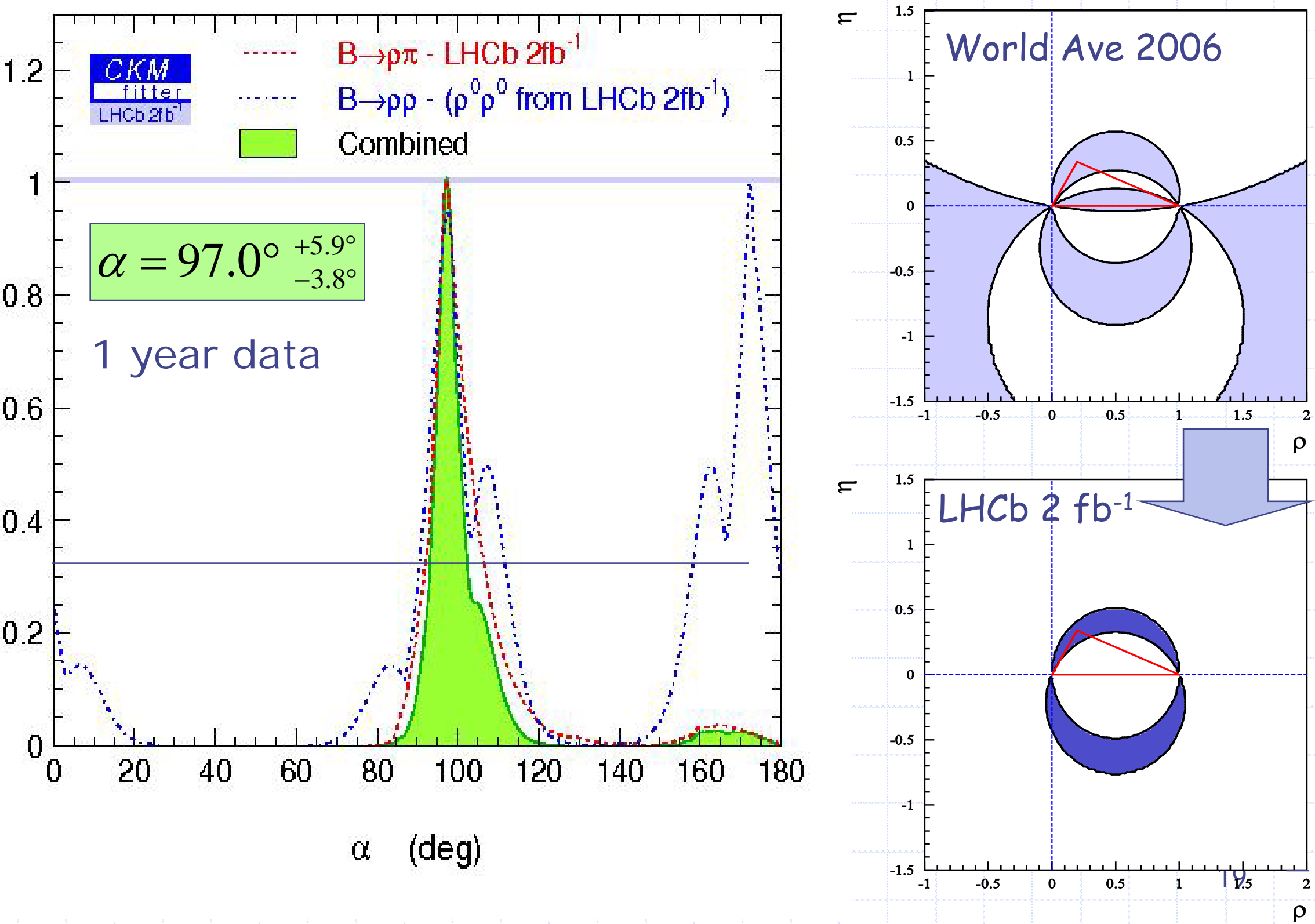


\section{Summary table}

\begin{tabular}{|c|c|c|c|c|c|}
\hline Angle & Channel & Yield* & $\mathbf{B}_{\mathrm{bb}} / \mathbf{S}$ & LHC ( $2 / \mathrm{fb})$ & $\begin{array}{c}\text { Theoretical } \\
\text { limit }\end{array}$ \\
\hline$\beta$ & $\begin{array}{l}\mathbf{B}_{\mathbf{d}} \rightarrow \mathbf{J} / \boldsymbol{\Psi} \mathbf{K}_{\mathbf{S}} \\
\mathbf{B}_{\mathbf{d}} \rightarrow \phi \mathbf{K}_{\mathbf{S}}\end{array}$ & $\begin{array}{l}216 k \\
0.8 k\end{array}$ & $\begin{array}{r}0.8 \\
<2.4\end{array}$ & $\begin{array}{l}\sigma(\beta) \approx 0.6^{\circ} \\
\sigma(\beta) \approx 12^{\circ}\end{array}$ & $\begin{array}{l}\sigma(\beta) \sim 0.2^{\circ} \\
\sigma(\beta) \sim 2^{\circ}\end{array}$ \\
\hline$\phi_{\mathrm{s}}$ & $\begin{array}{l}B_{s} \rightarrow J / \Psi \Phi \\
B_{s} \rightarrow J / \Psi \eta \\
B_{s} \rightarrow \eta_{c} \Phi\end{array}$ & $\begin{array}{c}125 k \\
12 k \\
3 k\end{array}$ & $\begin{array}{l}0.3 \\
2-3 \\
0.7\end{array}$ & $\sigma\left(\phi_{s}\right) \approx 1.2^{\circ}$ & $\sigma\left(\phi_{s}\right) \sim 0.2^{\circ}$ \\
\hline$\gamma$ & $\begin{array}{l}B_{s} \rightarrow D_{s} K \\
B_{d} \rightarrow \Pi \Pi \\
B_{s} \rightarrow K K \\
B_{d} \rightarrow D^{0}\left(K^{-} n^{+}\right) K^{* 0} \\
B_{d} \rightarrow D^{0}\left(K^{+} \Pi^{-}\right) K^{* 0} \\
B_{d} \rightarrow D_{c P}\left(K^{+} K^{-}\right) K^{* 0} \\
B^{-} \rightarrow D^{0}\left(K^{+} \Pi^{-}\right) K^{-} \\
B^{-} \rightarrow D^{0}\left(K^{-} n^{+}\right) K^{-}\end{array}$ & $\begin{array}{c}5.4 \mathrm{k} \\
26 \mathrm{k} \\
37 \mathrm{k} \\
0.5 \mathrm{k} \\
2.4 \mathrm{k} \\
0.6 \mathrm{k} \\
60 \mathrm{k} \\
2 \mathrm{k}\end{array}$ & $\begin{array}{r}<1.0 \\
<0.7 \\
0.3 \\
<0.3 \\
<2.0 \\
<0.3 \\
0.5 \\
0.5\end{array}$ & $\begin{array}{l}\sigma(\gamma) \approx 13^{\circ} \\
\sigma(\gamma) \approx 5^{\circ} \\
\sigma(\gamma) \approx 8^{\circ} \\
\sigma(\gamma) \approx 4^{\circ}=13^{\circ}\end{array}$ & $\begin{array}{c}\boldsymbol{\sigma}(\gamma) \ll \mathbf{1}^{\circ} \\
\text { if } U-\text { spin } \\
\text { symmetry } \\
\mathbf{-}\end{array}$ \\
\hline$\alpha$ & $\mathbf{B}_{\mathbf{d}} \rightarrow \pi \rho, \rho \rho$ & $14 k$ & 0.8 & $\sigma(\alpha)<10^{\circ}$ & $\sigma(\alpha) \sim 1^{\circ}$ \\
\hline
\end{tabular}

* Untagged annual yield after trigger 


\section{Conclusion}

$\diamond$ LHC experiments will allow to measure the Unitarity Triangle very precisely

$\checkmark$ loop processes in the flavour sector will probe high energy scale!

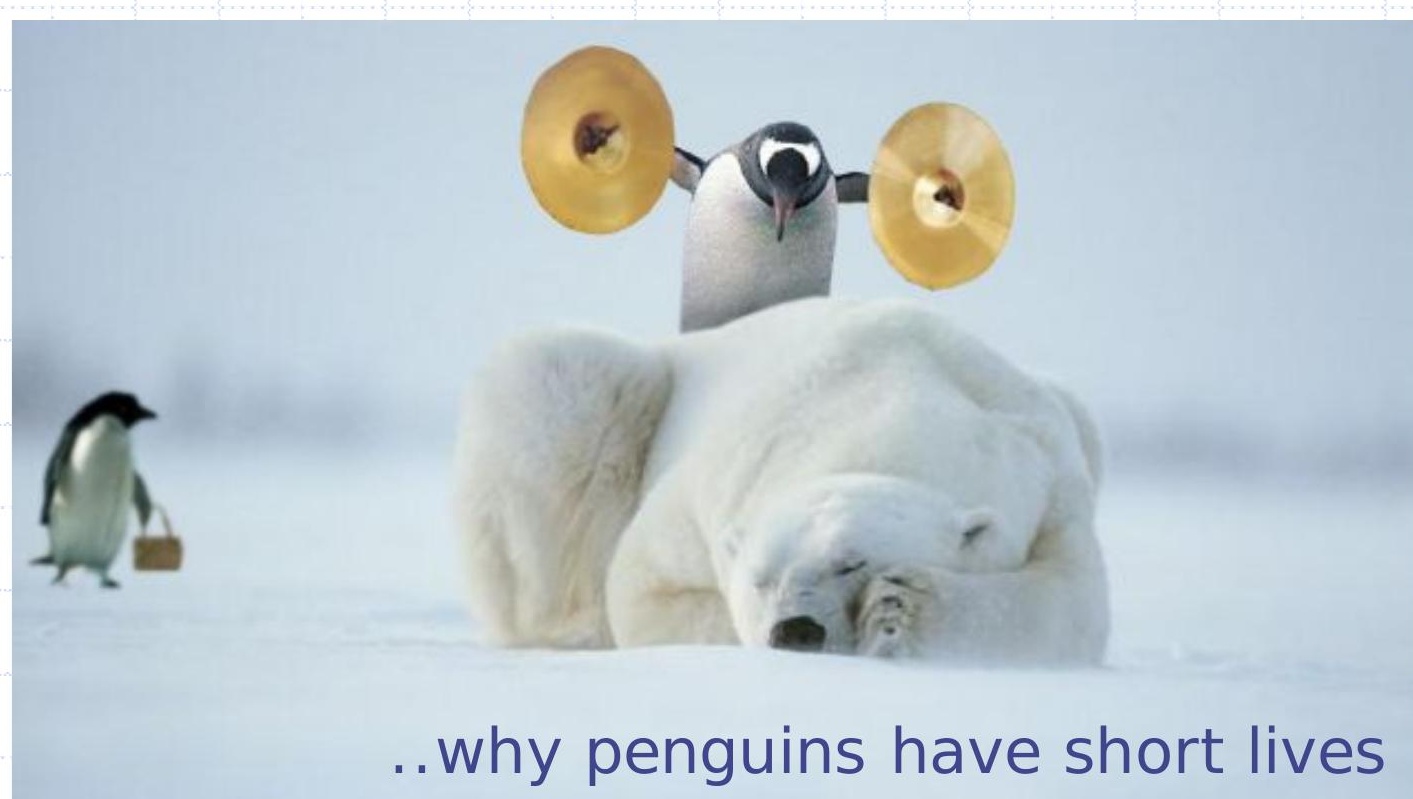

